\title{
Nanovolume Gas-Free Hydroxide Eluent Generator for Open Tubular Ion Chromatography
}

\author{
Bikash Chouhan, ${ }^{+}$Charles Phillip Shelor, ${ }^{\dagger}$ Weixiong Huang, ${ }^{\dagger \ddagger}$ Yongjing Chen, ${ }^{+₫}$ and Purnendu K. \\ Dasgupta $^{*+}$ \\ ${ }^{\dagger}$ Department of Chemistry and Biochemistry, University of Texas at Arlington, Arlington, Texas 76019-0065, United States. \\ ${ }^{\ddagger}$ School of Environmental Studies, China University of Geosciences, Wuhan, 430078, Hubei, China. ${ }^{\circledR}$ Thermo Fisher Scientific, \\ 1228 Titan Way, Sunnyvale, California 94088-3603, United States.
}

\section{Table of Content}

Figure S1. Experimental arrangement for determining the effect of the applied field strength $\quad$ S2

Figure S2. Photograph of the nanovolume eluent generator S3

$\begin{array}{ll}\text { Figure S3. Modifications made to the commercial cross fitting } & \text { S4 }\end{array}$

Figure S4. Single cross modification to make nanovolume EG without tees S5

Figure S5. Using the PSoC as a programmable current source S6

$\begin{array}{ll}\text { Table S1. Specific conductance data for } \mathrm{KOH} \text { solutions } & \mathrm{S7}\end{array}$

Figure S6. Specific conductance plot of $\mathrm{KOH}$ solutions with polynomial fit S8

Table S2. Calculated specific conductance data for 10-100 mM KOH solutions (ref. 40) S9

Figure S7. Specific resistance (bipolar pulse measurement of water at different field strengths S10

Figure S8. Illustrative outputs for triangular wave and long-duration square wave current ramps S11 


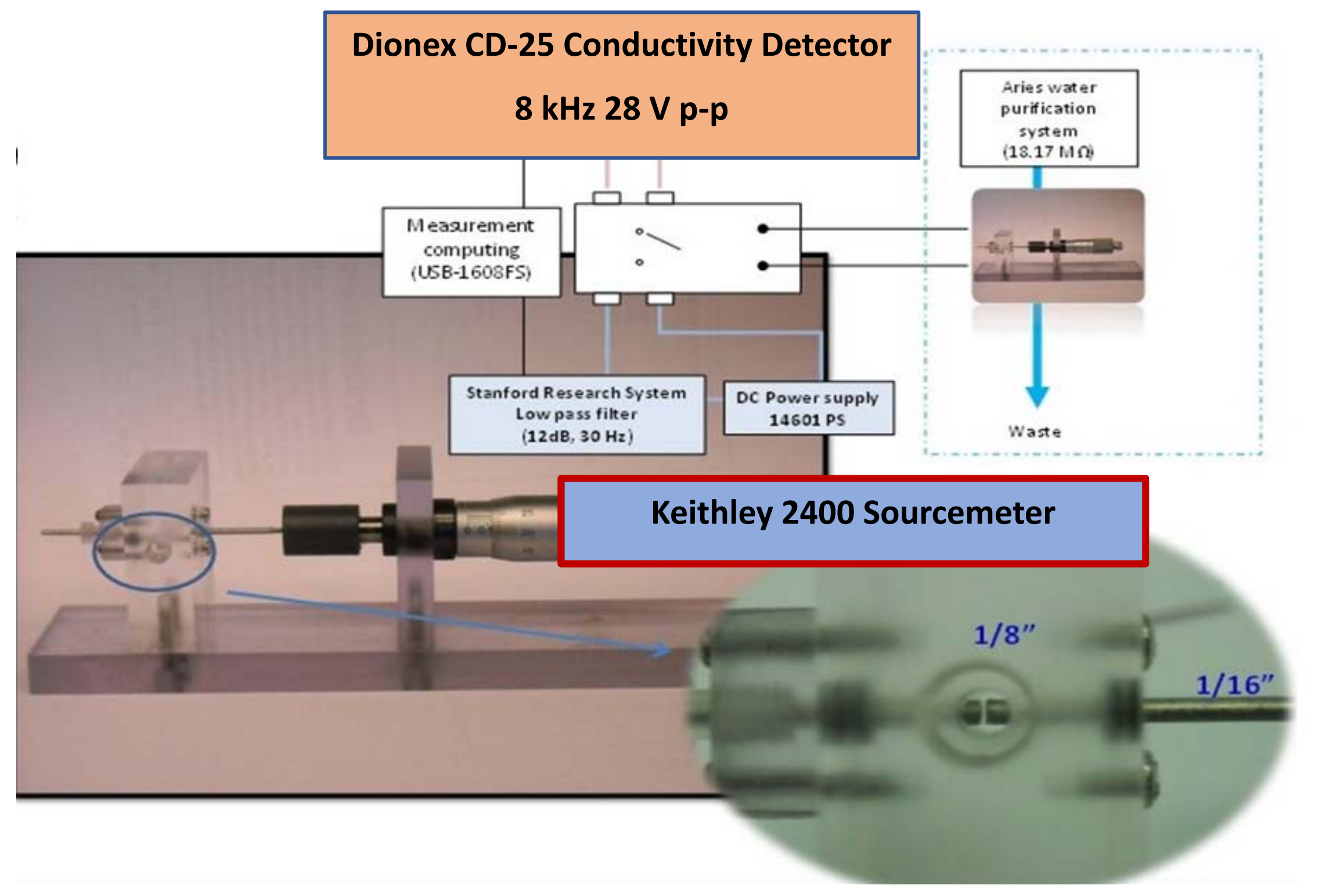

Figure S1. Experimental arrangement for determining the effect of the applied field strength photographically shown. Water passes horizontally through the $1 / 4-28$ threaded $1 / 8$ in bore flow path at $\sim 7 \mathrm{~mL} / \mathrm{min}$. While the electrodes are visible when the fittings to the bore are removed, note that the electrodes have transparent thin PTFE sleeves - only the faces are in contact with the fluid. A DPDT switch allows interrogation in the DC or bipolar pulse mode. 


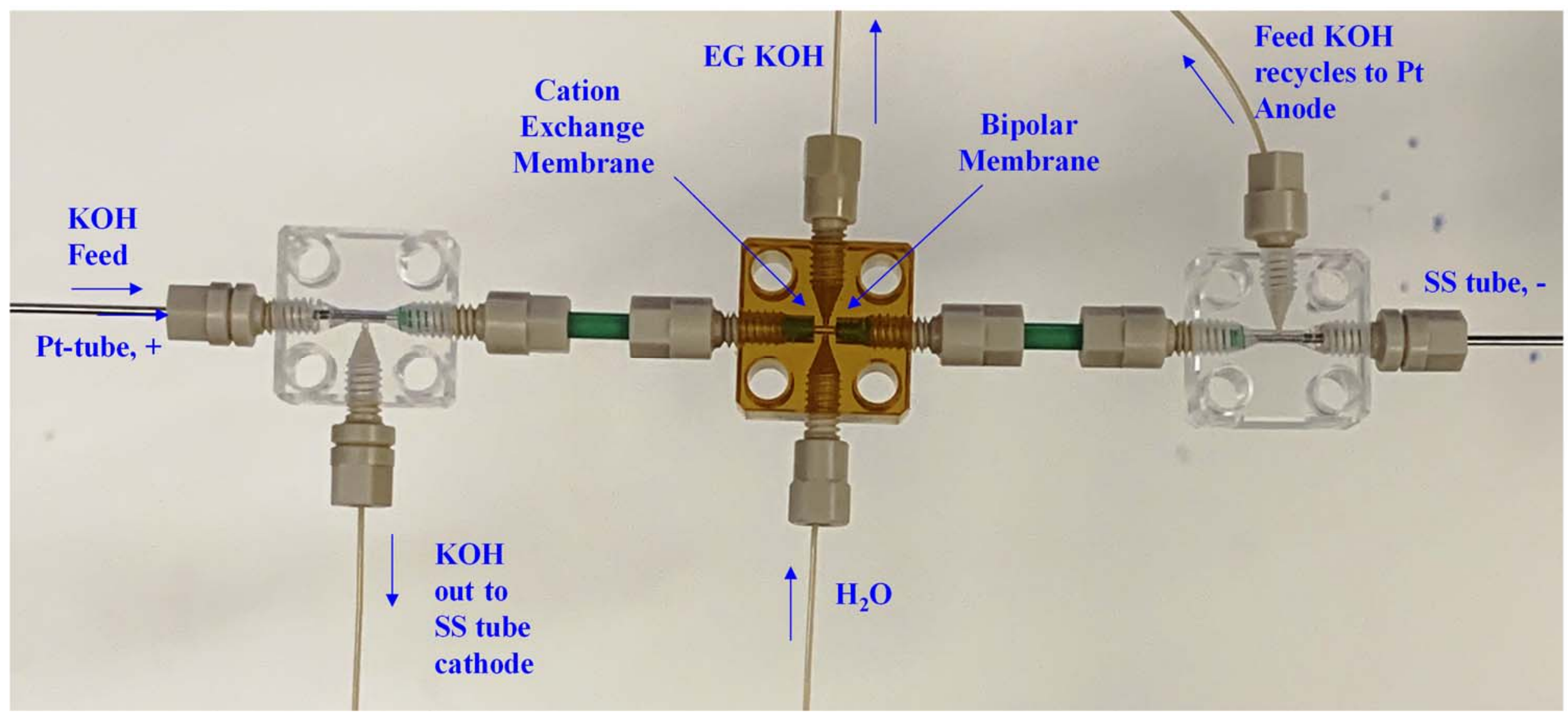

Figure S2. Photograph of the Nanovolume EG 


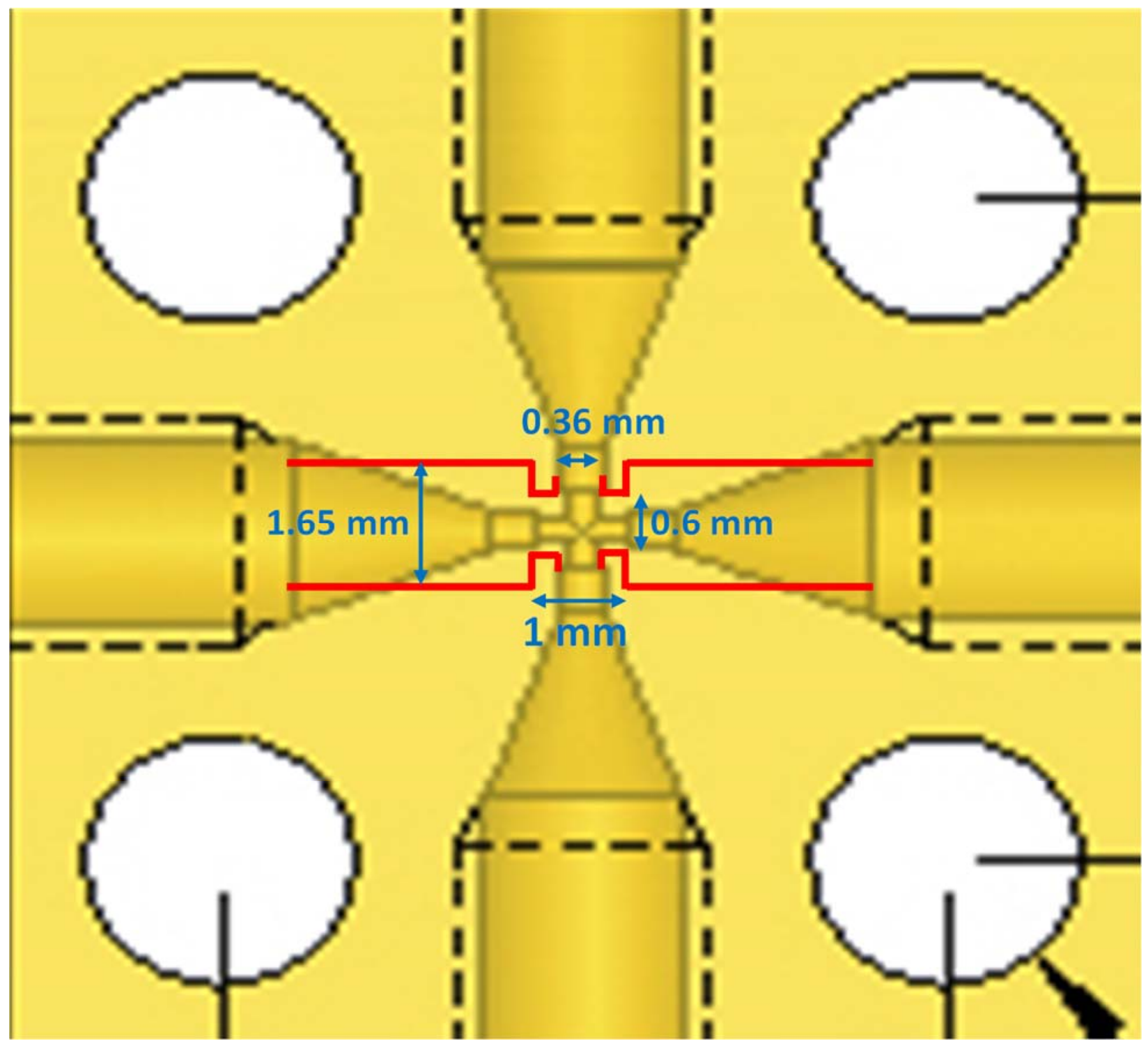

Figure S3. The red lines indicate modifications made to the commercial cross 

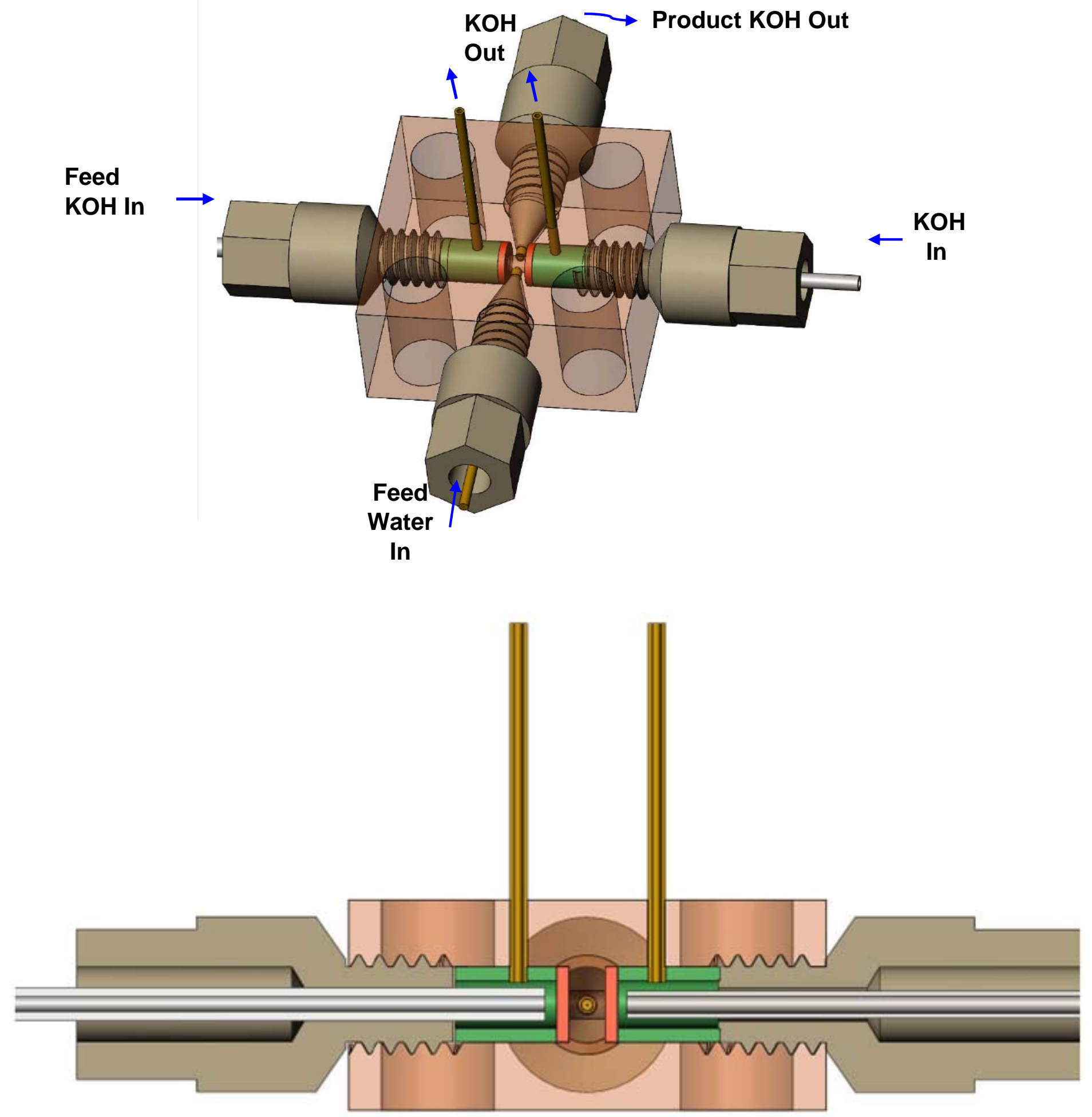

Figure S4. The nanovolume EG built on a single cross without need for further tees. The overall view is shown in the top figure where $360 \mu \mathrm{m}$ o.d. $150 \mu \mathrm{m}$ i.d. PEEK tubes access the compartments behind the membranes. The bottom figure shows the cutaway view from the front edge. 
Using PSoC 5LP as a Programmable Current Source. The PSoC 5LP programmable system on a chip is a versatile inexpensive (\$10) USB-powered device that has many attractive attributes (https://www.cypress.com/documentation/developmentkitsboards/cy8ckit-059-psoc-5lp-prototyping-kit-onboard-programmer-and).

It can be directly used as a current source using its digital to analog current (Current DAC) capability but with somewhat limited resolution. The current DAC has 8-bit resolution and permits operation in three ranges: 0-32, 0-255, and 0-2040 $\mu \mathrm{A}$. The respective (approximate) resolution is $0.125,1$, and $8 \mu \mathrm{A} / \mathrm{bit}$. It can be set to positive (source) or negative (sink) current. The range can be software programmed in a manner to both have the highest resolution in the low end and still use the full $2.04 \mathrm{~mA}$ capability, albeit for the present application, the current needs are not likely to exceed that provided by the lowest range. The main practical limitation that may arise about using the PSoC directly as the current source is its maximum voltage output capability of $5 \mathrm{~V}$, especially if the EG inner bore is limited to the original passage diameter of the cross.

\section{Using the PSoC Programmable Voltage Output DAC Through an Operational} Amplifier. The PSoC provides multiple 12-bit voltage DACs which can be used as current DACs as used in conjunction with one of the on-board operational amplifiers. The rest of the external circuitry follows an application note from Analog Devices (see Figure 2, Application Note CN-0151, https://www.analog.com/en/designcenter/reference-designs/circuits-from-the-lab/cn0151.html).

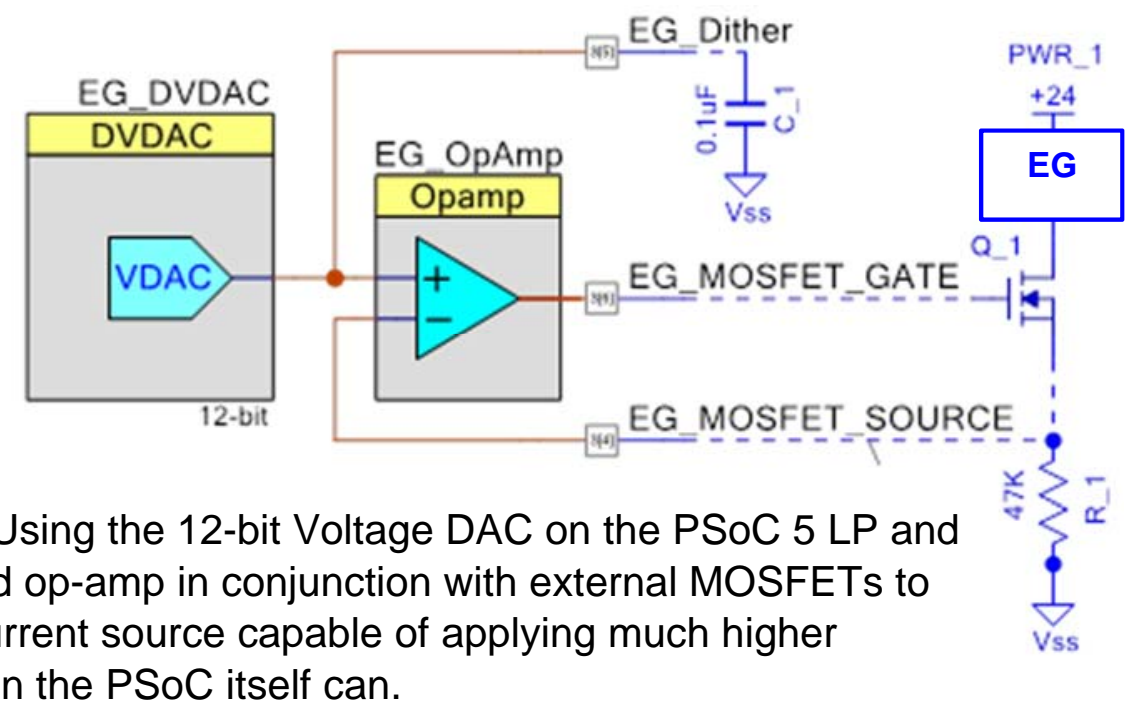

The analog voltage output from the PSoC) goes directly to one of the on-board op-amps with an external pin used for dithering the VDAC output to obtain 12-bit resolution. The op-amp is connected to an N-type MOSFET (BSH103, https://assets.nexperia.com/documents/data-sheet/BSH103.pdf) as shown. The controlled current is $I=V^{*} D / R 1$ where $V$ is the analog voltage output and $D$ is the 
fractional bit setting (actual bit setting/4095). R1, the current range setting resistor in this case is $47,000 \mathrm{ohms}$. With the maximum analog voltage output being $4.08 \mathrm{~V}$, the maximum current will be $4.08 / 47000=86.8 \mu \mathrm{A}$, controllable with 12-bit resolution. In the present application it is preferable to reduce maximum $\mathrm{V}_{\text {out }}$ to $1.02 \mathrm{~V}$ for higher resolution with a current ceiling of $21.7 \mu \mathrm{A}$ with a step resolution of $5.3 \mathrm{nA}$.

Specific Conductance Data for KOH. The following data for the KOH specific conductance of $\mathrm{KOH}$ at $25^{\circ} \mathrm{C}$ was supplied by Dr. Yan Liu, Thermo Fisher Scientific. Any departure from $25^{\circ} \mathrm{C}$ in the measurement was corrected using a temperature coefficient of $1.7 \% /{ }^{\circ} \mathrm{C}$. For the specific conductance for $0.1 \mathrm{mM} \mathrm{KOH}$, the tabulated infinite dilution equivalent conductance data for $\mathrm{K}^{+}$and $\mathrm{OH}^{-}$(see ref. 31 in main text) are assumed to be applicable.

\section{Table S1. Specific Conductance of KOH}

$\begin{array}{ccc}\begin{array}{c}\text { KOH } \\ \text { Concentration }\end{array} & \begin{array}{c}\text { Specific } \\ \text { Conductance }\end{array} & \begin{array}{c}\text { Polynomial } \\ \text { Prediction }\end{array} \\ \text { mM } & \mu \text { S/cm @ 25 C } & \mu \text { S/cm } \\ 0.1 & 27.21 & 29.13 \\ 1 & 271.2 & 267.9 \\ 2 & 530.2 & 529.7 \\ 3 & 786.2 & 788.3 \\ 4 & 1044 & 1044 \\ 10 & 2536 & 2536 \\ 20 & 4928 & 4928 \\ 30 & 7221 & 7221 \\ 50 & 11560 & 11560 \\ 80 & 17580 & 17600\end{array}$

alf actual temperature differed from $25^{\circ} \mathrm{C}$, this was corrected assuming a $1.7 \% /{ }^{\circ} \mathrm{C}$ temperature dependence of conductance. The best fit (four significant digit limited) $6^{\text {th }}$ order polynomial equation is given as

Specific Conductance $(\mu \mathrm{S} / \mathrm{cm})=2.391+267.6 c-2.146 c^{2}+9.648 \times 10^{-2} c^{3}-2.838 x$ $10-^{3} c^{4}+3.964 \times 10^{-5} c^{5}-2.027 \times 10^{-7} c^{6}$

where $c$ is the concentration of $\mathrm{KOH}$ is in $\mathrm{mM}$. A plot of the data and the polynomial best fit is shown overleaf as Figure S6.

A $\log (c, \mathrm{mM})$ vs. $\log ($ specific conductance, $\mu \mathrm{S} / \mathrm{cm})$ plot has the best fit relationship

$\log ($ specific conductance, $\mu \mathrm{S} / \mathrm{cm})=0.9684 * \log (c, \mathrm{mM})+2.425$ 
This much simpler equation fits all the data except that at the lowest and highest concentration with less than $2.5 \%$ error and may be acceptable for most purposes. The specific \% errors of prediction, from lowest to highest concentration, respectively, are: $5.2,-1.9,-1.8,-1.9,-2.4,-2.4,-1.8,-0.7,1.7,5.4 \%$.

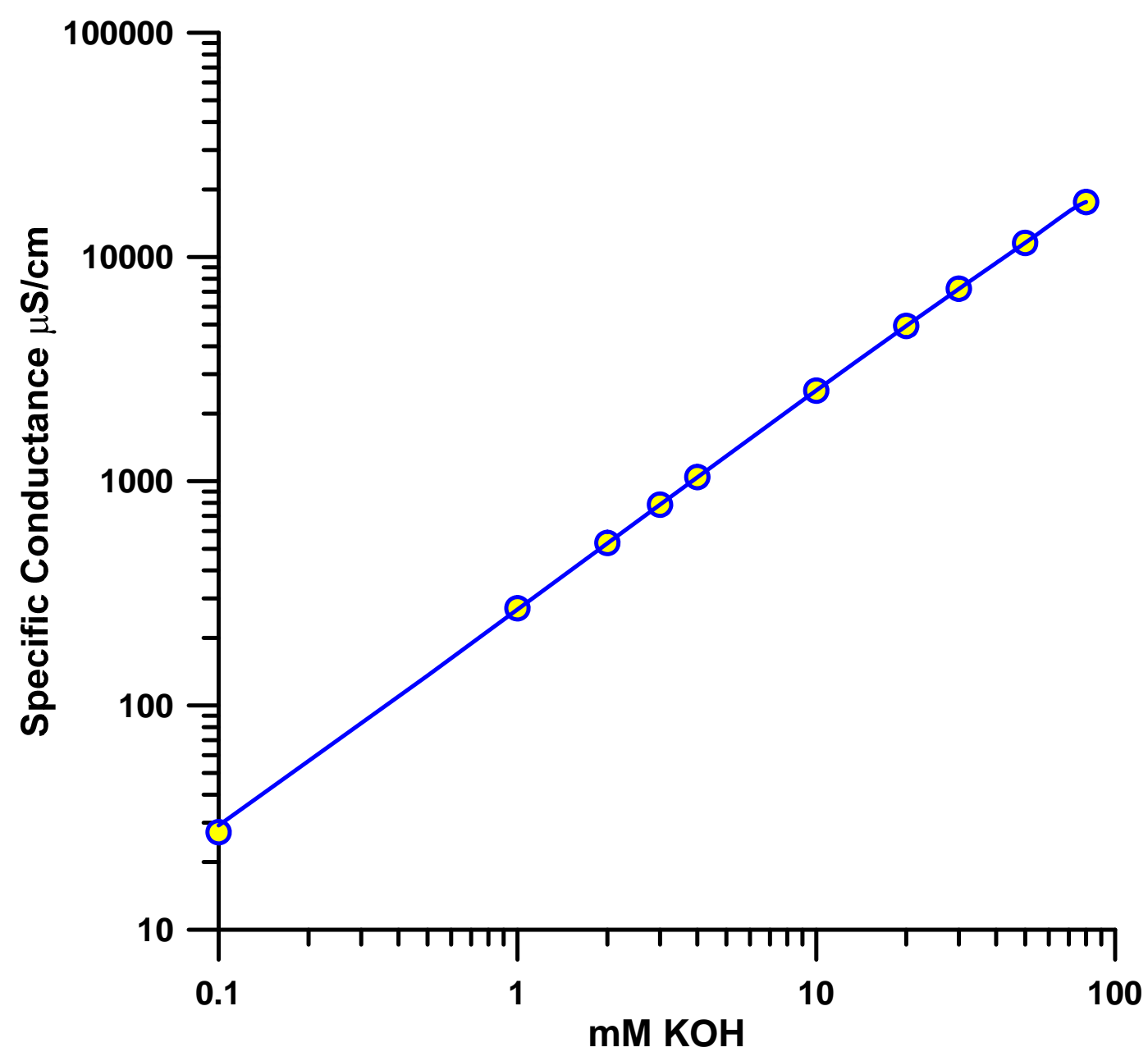

Figure S6. The specific conductance of $\mathrm{KOH}$ solutions. The blueline is the $6^{\text {th }}$ order polynomial fit for the data in the first two columns in Table S1; the values are in col. 3.

Gilliam et al. (main text, ref. 43) provides calculated values of $\mathrm{KOH}$ specific conductance at various temperatures for $10,20,30,50$, and $100 \mathrm{mM} \mathrm{KOH}$ (and higher concentrations) based on a literature review of data that are generally at much higher concentrations. Throughout the above concentration range, the temperature coefficient for the specific conductance of $\mathrm{KOH}$ from their calculated values is in the range of 1.2$1.3 \%$. Values for $10-50 \mathrm{mM}$ are within $5 \%$ of the values in Table S1. 
Table S2. Calculated KOH Specific Conductance $(\mu \mathrm{S} / \mathrm{cm})^{\mathrm{a}}$

$\begin{array}{cccc}\text { mM } & \mathbf{2 0 ~ C} & \mathbf{2 5 C} & \mathbf{3 0 ~ C} \\ \text { KOH } & & & \\ 10 & 2290 & 2430 & 2590 \\ 20 & 4570 & 4860 & 5170 \\ 30 & 6840 & 7280 & 7740 \\ 50 & 11400 & 12100 & 12900 \\ 100 & 22600 & 24100 & 25600\end{array}$

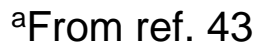




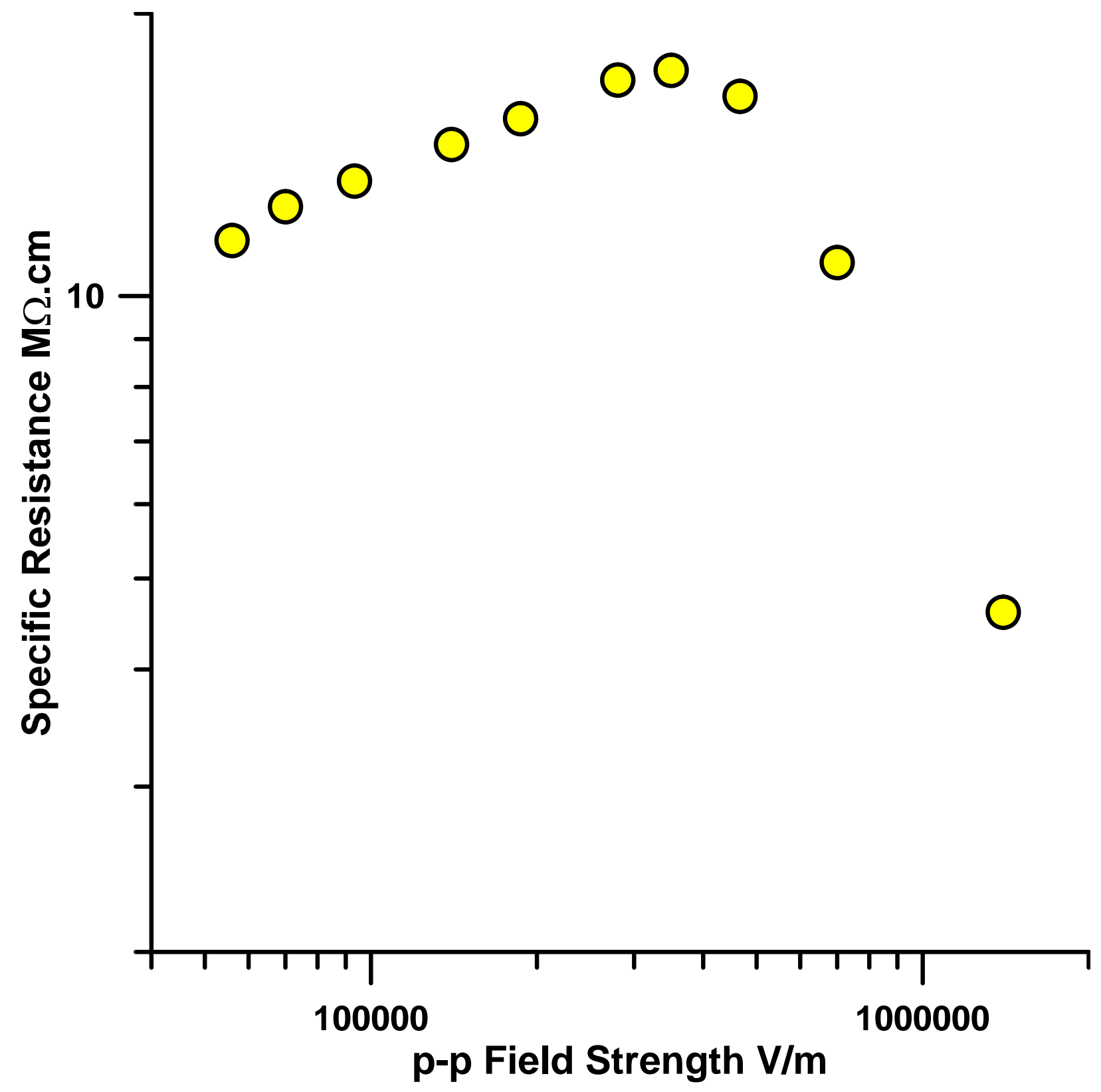

Figure S7. Specific resistance of pure water as measured with a Dionex CD25 bipolar pulse conductance detector. The applied voltage was fixed at $28 \mathrm{~V} \mathrm{p-p;} \mathrm{the} \mathrm{field}$ strength was varied as the interelectrode distance was changed. There is uncertainty related to absolute accuracy of the electrode distance and the parallelism of the electrode faces but at lower fields the measured resistivity remains within 12-17 M $2 . \mathrm{cm}$ and starts decreasing abruptly at field strengths $\geq 4 \times 10^{5} \mathrm{~V} / \mathrm{m}$. The difference between $\mathrm{DC}$ and $\mathrm{AC}$ measurements may lie in the finite time needed for ion atmosphere formation. 


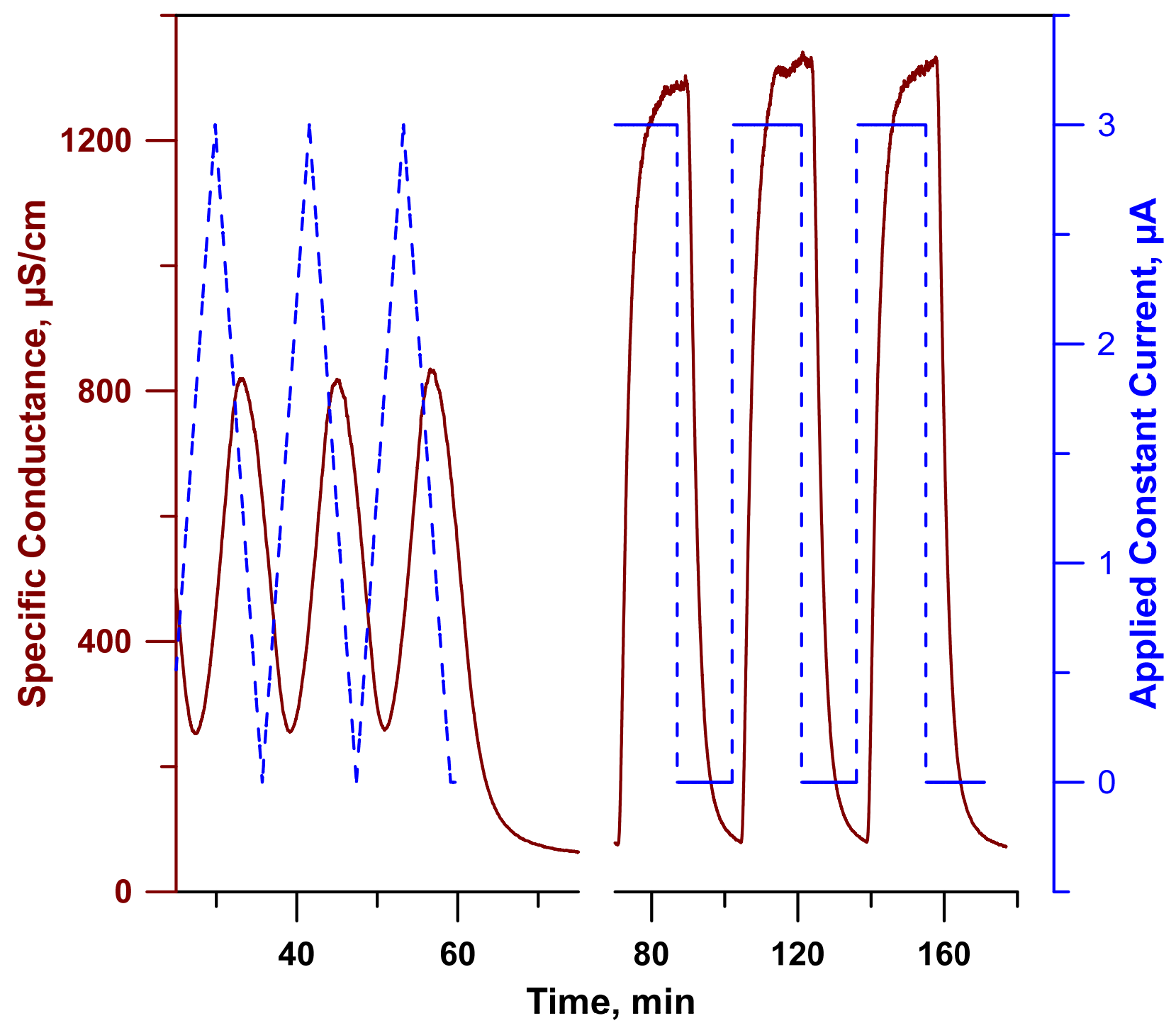

Figure S8. Illustrative generator output with triangular wave (flow rate $180 \mathrm{~nL} / \mathrm{min}$ ) and square wave current ramps (flow rate $172 \mathrm{~nL} / \mathrm{min}$ ). The detector was located $30 \mathrm{~cm}$ from the EG, essentially mid-column for typical column lengths of $60 \mathrm{~cm}$. Note the phase lag between the applied ramp and observed conductance. Nevertheless, for the most relevant part, the upward ramp in the triangular wave part, the conductance ascent is virtually parallel to the current ascent, the steepness of the slope is not sacrificed. 